\title{
Análise de Sustentabilidade dos Sistemas Microgrids de Geração de Energia Elétrica na Amazônia: Estudo de Caso em Rondônia
}

\author{
Cristiano Torres do Amaral* \\ Universidade Federal de Minas Gerais
}

\section{Artur de Souza Moret*}

Universidade Federal de Rondônia

\begin{abstract}
Resumo: Este trabalho tem por objetivo demonstrar a metodologia estatística adequada para análise da sustentabilidade dos sistemas microgrids, tradução em inglês de microrredes e utilizado como termo para se referir a geração de energia em áreas isoladas, em especial, no estado de Rondônia. Neste estudo foi realizada breve revisão e descrição atualizada da rede de geração e distribuição de energia elétrica no estado de Rondônia, bem como respectivo levantamento estatístico e mapeamento das unidades em operação. Em seguida, foram aplicados indicadores de desempenho social, econômico e ambiental para compor uma matriz de sustentabilidade dos sistemas microgrids. A matriz de sustentabilidade demonstra graficamente os critérios e requisitos de sustentabilidade que pode orientar investimentos de empreendedores, e ainda, gestores públicos na elaboração de políticas de incentivo, implantação e regulação dos sistemas microgrids no estado de Rondônia.
\end{abstract}

Palavras chave: energia limpa; energia renovável; desenvolvimento sustentável.

\section{SUSTAINABILITY ANALYSIS OF THE MICROGRIDS SYSTEMS OF ELECTRIC POWER GENERATION IN THE AMAZON: CASE STUDY IN RONDÔNIA}

Abstract: The aim of this work is to demonstrate the adequate statistical methodology for the analysis of the sustainability of microgrids systems, the English translation of microgrids is used as a term to refer to the generation of energy in isolated areas, especially in the state of Rondônia. In this study, a brief review and updated description of the electricity generation and distribution network in the state of Rondônia was carried out, as well as a statistical survey and mapping of the units in operation. Afterwards, indicators of social, economic and environmental performance were applied to compose a sustainability matrix of microgrids systems. The sustainability matrix graphically demonstrates the criteria and sustainability requirements that can guide investments by entrepreneurs and public managers in the elaboration of incentive, implementation and regulation policies for microgrids systems in the state of Rondônia.

Keywords: clean energy; renewable energy; sustainable development.

\section{ANÁLISIS DE SOSTENIBILIDAD DE LOS SISTEMAS MICROGRIDS PARA GENERACIÓN DE ELECTRICIDAD EN LA AMAZONÍA: UN ESTUDIO DE CASO EN RONDÔNIA}

El objetivo de este trabajo es demostrar la metodología estadística adecuada para análisis de la sostenibilidad de sistemas microgrids, la traducción al inglés de las micro redes, y su uso como un término para referirse a la generación de energía en áreas aisladas, especialmente en el estado de Rondônia. En este estudio, se realizó una breve revisión que describió la distribución de energía y la distribución de electricidad en el estado de Rondônia, así como la encuesta estadística y el mapeo de las unidades en operación. Luego, se seleccionaron indicadores de desempeño social, económico y ambiental para componer una matriz de sostenibilidad para sistemas de micro redes. Una matriz de sostenibilidad muestra gráficamente los requisitos de sostenibilidad y los requisitos que pueden guiar las inversiones de los empresarios, así como de los administradores públicos, utilizando políticas públicas de políticas de incentivos, implantación y aplicación de sistemas de microrredes en el estado de Rondônia.

Palabras clave: energía limpia; energía renovable; desarrollo sostenible. gov.br

${ }_{*}^{*}$ Doutor em Planejamento de Sistemas Energéticos. Coordenador do Programa de Pós-Graduação em Desenvolvimento Regional e Meio Ambiente da Fundação Universidade Federal de Rondônia. Endereço: BR 364 Km 9,5, CEP. 76800-000, Porto Velho - RO. ORCID:https://orcid.org/0000-0001-7371-5486 E-mail: amoret@unir.br 
Introdução

O modo de produção e o padrão atual de consumo comprometem as condições de sobrevivência da humanidade e do meio ambiente, destruindo e degradando ecossistemas e os recursos naturais. Todos os anos mais pessoas se inserem no mercado global e aumenta a demanda por recursos naturais. Os mercados que não estão incluídos pressionam pela oferta de matéria-prima e infraestrutura, principalmente de energia elétrica. No Brasil, existem muitas comunidades que não tem acesso aos serviços de energia elétrica, deixando inúmeras pessoas excluídas, a margem do desenvolvimento. De acordo com levantamento da Pesquisa Nacional por Amostra de Domicílios (PNAD), $99,6 \%$ da população tem acesso à energia elétrica e $0,4 \%$ da população, cerca de 800.000 pessoas, ainda não são atendidas pela rede distribuição de energia em todo o país (MME, 2016).

O sistema capitalista tem como premissa a expansão do capital, independente das limitações do sistema, não respeita as restrições do espaço e a produção sempre busca metas de crescimento maiores em um planeta finito (HARVEY, 2003). Em um movimento oposto, em contraposição ao capitalismo, o desenvolvimento sustentável se apresenta como resposta a crise imposta pelo capitalismo, tentando corrigir problemas ambientais, sociais e éticos, propondo a produção e o consumo baseados no equilíbrio entre o homem e a natureza.

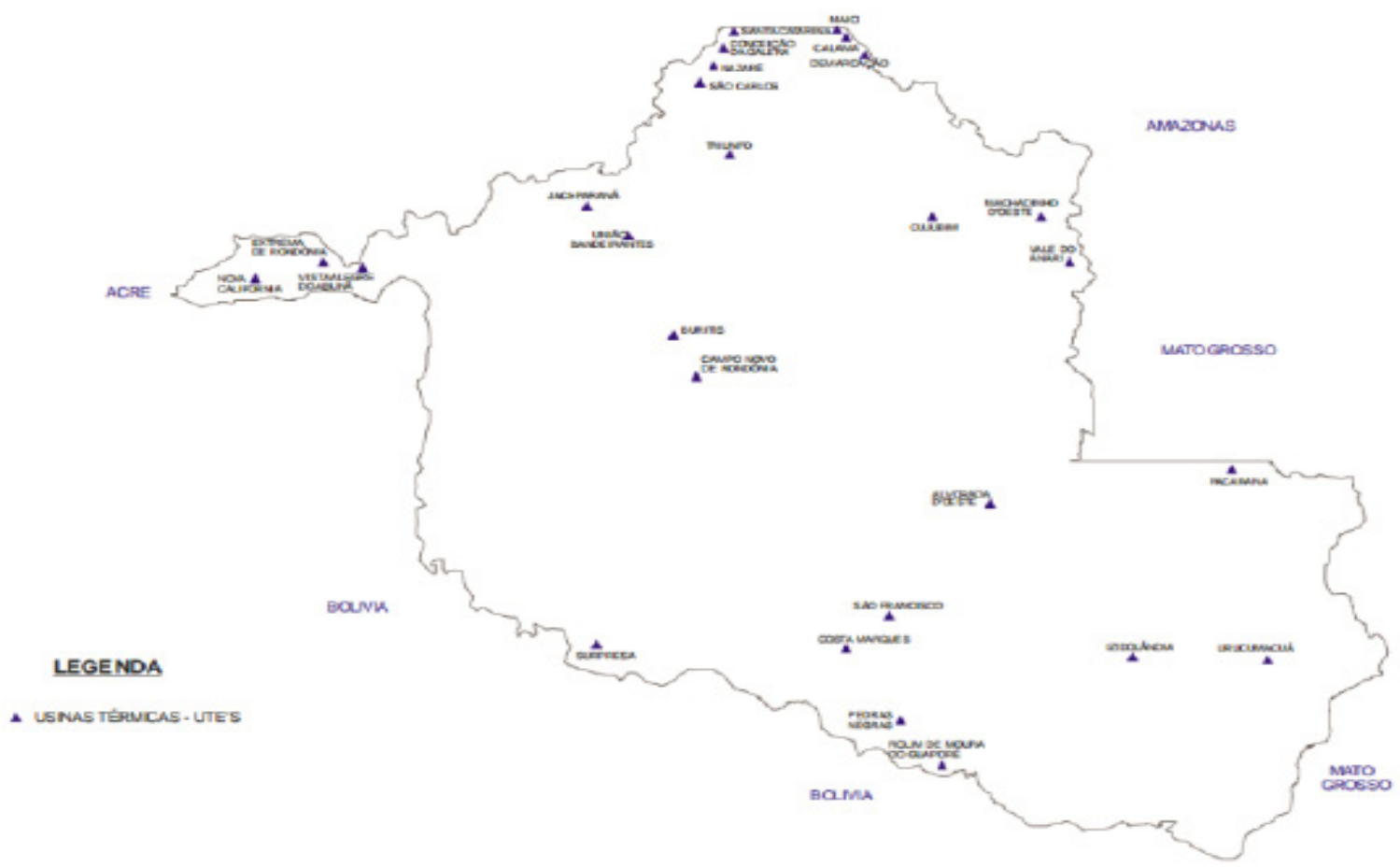

Fonte: Adaptado de CERON/ELETROBRAS (2016) 
Em Rondônia, para atender a demanda de energia elétrica, a matriz energética do estado foi estruturada a partir de um sistema hidro-térmico interligado por quatro usinas hidrelétricas - UHE (Samuel, Santo Antônio, Jirau, Rondon II), 2 usinas térmicas - UTE (Termo Norte e Eletronorte), 17 Pequenas Centrais Hidrelétricas (PCH) e 26 sistemas isolados, minirredes - microgrids (Figura 1) que utilizam motores a diesel, sem conexão ao Sistema Interligado Nacional (SIN). Toda essa carga totaliza 80 empreendimentos em operação gerando $6.726 .396 \mathrm{~kW}$ (ANEEL, 2016a). O SIN atende cerca de $80 \%$ da demanda residencial. As microgrids dos isolados atendem cerca de 40 localidades no estado de Rondônia e seu consumo foi estimado em cerca de 82,3 milhões de litros de Diesel para geração de 306.001 MWh (ELETROBRAS, 2016).

Neste modelo de geração e distribuição, segundo Tolmasquim (2016), a universalização convencional da energia elétrica na Amazônia tem custo elevado e poderá exigir investimentos de até 8 bilhões de reais para atender todas as localidades em área urbana e rural. Devidos aos custos elevados e a necessidade de adotar práticas sustentáveis, é importante fomentar, por meio de programas e legislação específica, sistemas microgrids de geração híbrida, associando diferentes fontes de energia sustentáveis.

Logo, este trabalho aplica um modelo de medida de sustentabilidade aos empreendimentos de geração de energia no sistema microgrids isolados do estado de Rondônia, mensurando a sustentabilidade de cada empreendimento. Avaliando o nível de desenvolvimento sustentável de cada microgrid é possível identificar a sua contribuição no âmbito social, econômico e ambiental. Essa análise pode alicerçar o planejamento para futuros investimentos, orientando e priorizando práticas sustentáveis para os sistemas que possuem os indicadores de sustentabilidade desfavoráveis. Existem ferramentas de mensuração de sustentabilidade em desenvolvimento, entretanto, não são aplicáveis na Amazônia brasileira, bem como nos sistemas microgrids do sistema isolado de Rondônia.

\section{O setor elétrico em Rondônia}

O antigo Território Federal do Guaporé tinha o Serviço de Abastecimento de Água Luz e Força (SAALFT) como responsável pelo fornecimento de energia elétrica até o final dos anos 60. Em 1968 foi criada a empresa de economia mista Centrais Elétricas de Rondônia S.A. (CERON) que passa a cumprir a missão do SAALFT. Nos primeiros anos de fornecimento, a rede de geração e distribuição de energia elétrica era baseada em grupos geradores a diesel. Em 1981, no recém-criado Estado de Rondônia, as Centrais Elétricas do Norte do Brasil (Eletronorte) assumem o fornecimento da capital, Porto Velho. Em janeiro de 1987, foi inaugurada a primeira Pequena Central Hidrelétrica $(\mathrm{PCH})$ no município de Vilhena. Entretanto, desde aquele período, o fornecimento se manteve apoiado em usinas térmicas a diesel. Em julho de 1989, a primeira unidade da Usina Hidrelétrica Samuel, no Rio Jamari, entrou em operação (MORET, 2014).

Ao longo dos anos 90, no interior do Estado de Rondônia existiam cerca de 72 usinas térmicas operando em áreas isoladas. Atualmente, existem 25 sistemas isolados de geração de energia, minirredes (microgrids) sem conexão ao SIN, que atendem cerca de 40 localidades isoladas CERON/ELETROBRAS (2016). A energia bruta requerida ( $\mathrm{MWh}$ ) do sistema está disposto

Figura 2 - Energia Bruta Requerida em Rondônia (MWh)

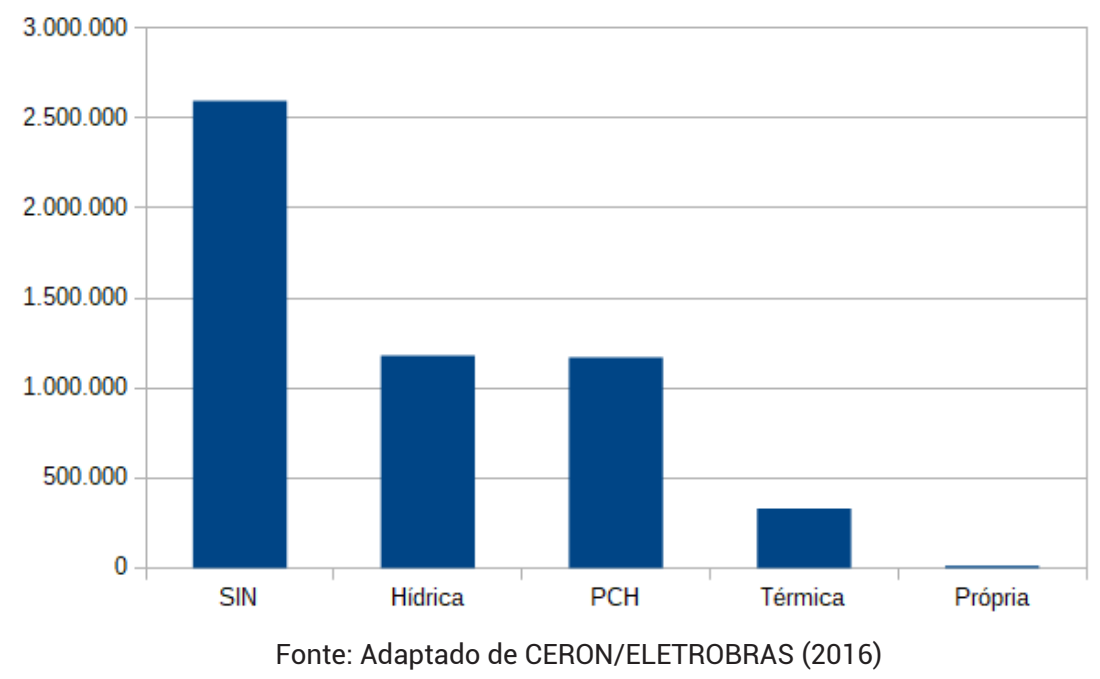


Figura 3 - Perfil dos Consumidores em Rondônia (Unidades)

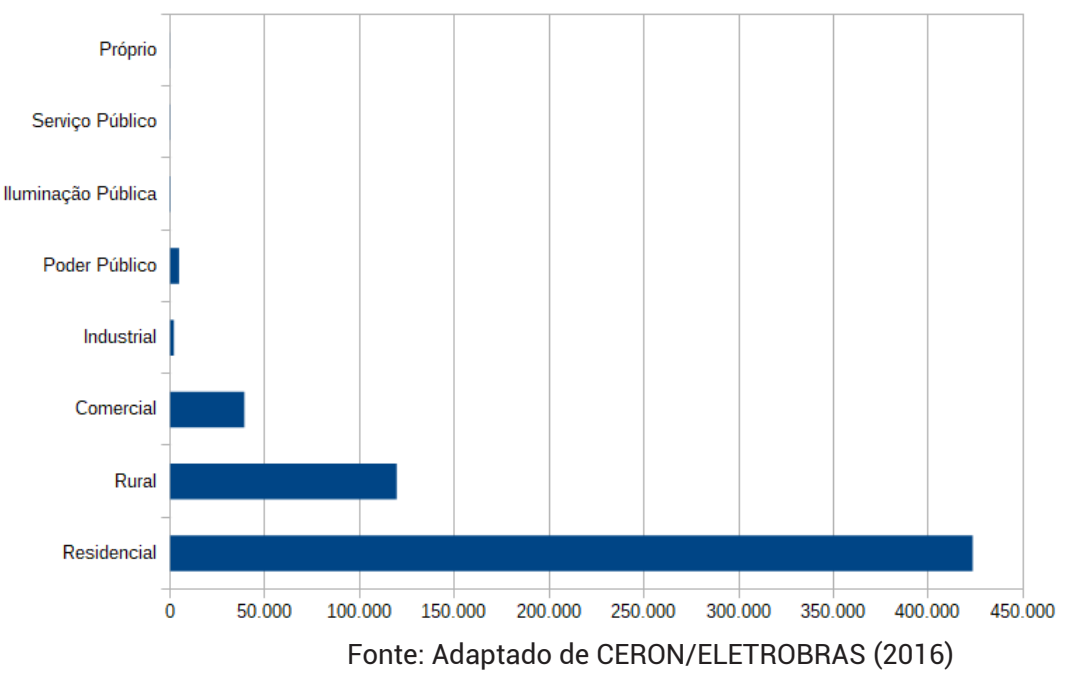

na Figura 2, a seguir, que apresenta cerca de $80 \%$ da

Figura 4 - Consumo Anual de Energia por Classe em Rondônia (MWh)

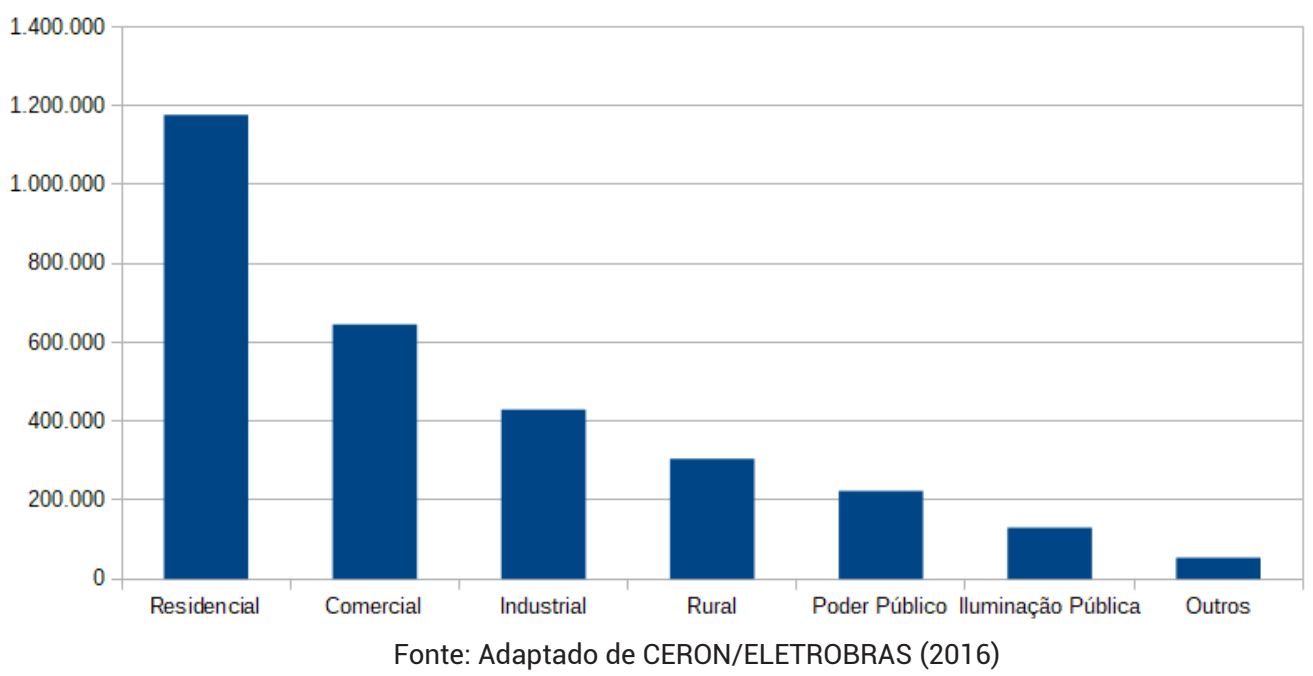

Figura 5 - Maiores Municípios Consumidores em Rondônia (MWh)
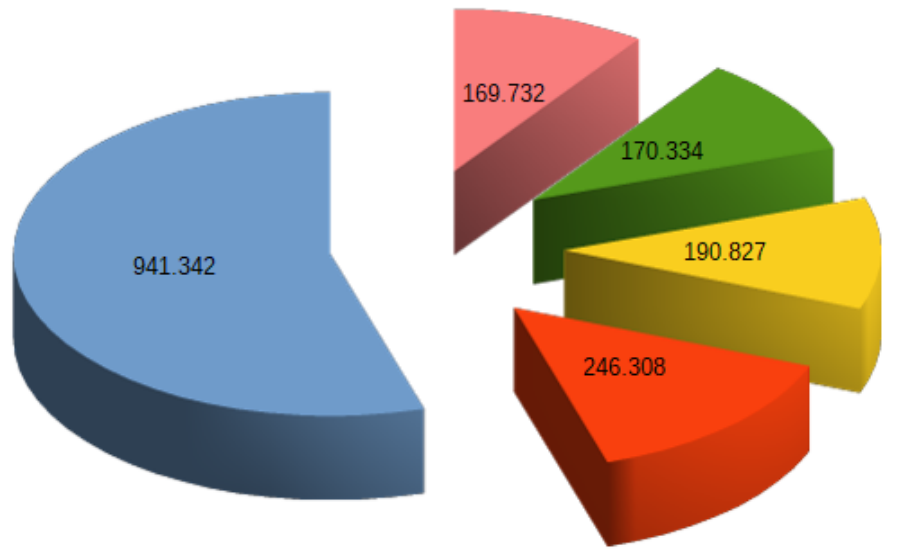

- Porto Velho

- Ji-Paraná

Ariquemes

- Vilhena

- Cacoal 
demanda atendida pelo SIN e, cerca $10 \%$ é ofertado pelas usinas térmicas do estado. A maior parte dessa demanda é destinada aos consumidores residenciais (Figuras 3 e 4) e ao município de Porto Velho (Figura 5), capital do estado.

A eletricidade anual consumida por todo o sistema isolado de Rondônia, conforme observado na Figura 2, alcança o montante de cerca de $335,6 \mathrm{GWh}$, o que representa apenas $6,2 \%$ do total da energia bruta requerida. Por sua vez, a usina hidrelétrica de Santo Antônio, em Porto Velho/RO tem potência instalada de 3.568 MWe pode gerar até 31,2 TWh funcionando 24 horas por dia em 365 dias do ano. A energia anual consumida pelo sistema isolado de Rondônia representa pouco mais de $1,07 \%$ de toda a energia anual gerada em Santo Antônio e poderia facilmente ser suprida pelo parque hidrelétrico instalado. Entretanto, os investimentos em linhas de distribuição não são suficientes para atender essa demanda. A rede de distribuição atende apenas as cidades ao longo do eixo da BR 364.

\section{Ferramentas de medida de sustentabilidade}

Avaliar a sustentabilidade de empreendimentos é muito importante e, no meio empresarial, as ferramentas mais comuns de análise da sustentabilidade estão associadas às recomendações e normas internacionais. Esta documentação orienta comportamentos e ações de caráter ambiental e social nas corporações. A norma ISO - International Organization for Standardization 14.001, por exemplo, estabelece as diretrizes para a instalação, operação e manutenção de um sistema de gestão ambiental. A ISO 14.001 utiliza a abordagem PDCA (do inglês: Plan, Do, Check e Act) e prevê ainda a elaboração de uma política ambiental, monitoramento e auditorias. Também existem as ISO 26.000 e SA 8.000 da Social Accountability International que estão relacionadas com a avaliação da responsabilidade social das empresas. A norma ISO 14.040 estabelece a Avaliação do Ciclo de Vida de um produto ou serviço e busca quantificar as emissões ambientais e avaliar o impacto no meio ambiente da cadeia produtiva (REIS, 2011).

Essas ferramentas são importantes e, algumas delas, também utilizam aplicações computacionais para estabelecer um índice global de sustentabilidade para uma determinada empresa, produto ou serviço. A Empresa BASF, por exemplo, comercializa o software AgBalance para mensurar a sustentabilidade na agricultura. Esta ferramenta coleta os dados da cadeia produtiva, processa as informações a partir de indicadores ponderados e apresenta os relatórios de sustentabilidade (BASF, 2016).

Estes softwares utilizam arquiteturas e modelos de medição de desempenho empresarial adaptados para desenvolver estratégias de sustentabilidade. Entre estes softwares de negócio se destacam o Strategic Measurement and Reporting Technique, Matriz de Medição de desempenho, Balanced Scorecard (BSC), Sistema de Medição de Desempenho de Cambridge (KAPLAN; NORTON, 1997); (HUBBARD, 2009).

Os indicadores de sustentabilidade alimentam as ferramentas de gestão e orientam os stakeholders na solução de questões que comprometam a sustentabilidade (FREEMAN, 1984). Estas ferramentas monitoram indicadores de desempenho e demonstram de maneira gráfica, ou em relatórios, a performance de uma organização para cada tema de sustentabilidade. Os sistemas híbridos de geração de energia não possuem metodologia de avaliação de sustentabilidade próprio, uma vez que exige temas e indicadores específicos para descrever um sistema multidisciplinar e complexo (OSTROM, 2009). Afgana, Carvalhoa e Hovanovb (2000) e Hatziargyriou, Vasiljevska e Tsikalakis (2009) apresentaram estudos para valorar, quantificar e estimar os benefícios da operação dos sistemas microgrids em áreas isoladas utilizando ferramentas numéricas e dados de comunidades localizadas em ilhas oceânicas.

Liu (2014), Lillo et al. (2015) e Slough, Urpelainen e Yang (2015) acrescentam que, no caso de sistemas de geração e distribuição em áreas isoladas, os temas e indicadores de sustentabilidade devem incorporar o crescimento econômico, e ainda, expressar a eficiência, equidade e qualidade de vida dos atores que estão inseridos no empreendimento do setor elétrico. Dessa maneira, os temas e indicadores de sustentabilidade podem demonstrar com maior clareza o uso racional dos recursos naturais para geração e distribuição de energia elétrica em uma comunidade.

\section{Materiais e Métodos}

A medição do desempenho de sustentabilidade dos sistemas microgrids consiste em quantificar a eficiência e eficácia do processo de operação e funcionamento. Neste estudo, a metodologia de análise de desempenho dos sistemas microgrids não utiliza apenas indicadores econômicos, mas correlaciona indicadores que descrevem os sistemas na dimensão ambiental, social e econômica, de maneira interdisciplinar, envolvendo todos os stakeholders e sua complexidade (OSTROM, 
2009).

Os sistemas microgrid estão instalados em 25 localidades isoladas no estado de Rondônia e são nessas áreas que vivem as comunidades mais carentes (CERON/ELETROBRAS, 2016). Promover a instalação de sistemas de geração de energia eficientes, com fontes limpas e renováveis é de fundamental importância para promover o desenvolvimento dessas comunidades respeitando o meio ambiente (MORET, 2014).

A amostra contempla os sistemas microgrids em operação na Amazônia e, por isso, abrange os sistemas microgrids de geração isolados em Rondônia e que adotam Produção Independente de Energia (PIE) para $100 \%$ de uso local que utilizam combustíveis fósseis, contemplando as 25 localidades onde estão instalados (LIU, 2014).

Inicialmente é considerada a sustentabilidade dos sistemas microgrids instalados no estado $\left(H_{0}\right)$ (MONTGOMERY, 2012). 0 modelo de sustentabilidade deverá testar a hipótese nula $\left(H_{0}\right)$, verificando uma hipótese alternativa $\left(H_{1}\right)$ que considera os sistemas microgrids insustentáveis para cada tema proposto, bem como classificando-os em três níveis de sustentabilidade (LIU, 2014).

A abordagem descritiva será utilizada para identificação, registro e análise das características dos sistemas microgrids, bem como fatores ou variáveis que se relacionam com a sua sustentabilidade. A sustentabilidade será avaliada a partir de uma matriz com indicadores ambientais, sociais e econômicos. Os dados utilizados para determinação dos indicadores de sustentabilidade serão obtidos por meio de pesquisa nas bases de dados oficiais dos principais atores envolvidos: unidades gestoras de microgrids, concessionária de energia, prestadores de serviço, fornecedores e unidades consumidoras (OSTROM, 2009).

De acordo com Liu (2014) e Yadoo e Cruickshank (2012), um indicador de sustentabilidade em sistemas microgrids precisa descrever diferentes dimensões sociais, econômicas e ambientais, auxiliando na gestão e indicando a sua proximidade com a realidade. Os indicadores são parâmetros representativos dos processos e que possibilitam quantificá-los em relação aos dados da amostra analisada para comparação dos níveis de sustentabilidade dos sistemas microgrids.

Nesta pesquisa os indicadores são balizados pelos temas de sustentabilidade propostos por Nascimento (2012) e abordam a relação ambiental, econômica, social de um sistema microgrid. Nesta etapa de seleção de temas e indicadores foram utilizadas as premissas propostas por Liu (2014).

Em seguida, conforme recomendado por Liu (2014), os dados apurados foram classificados em 3 níveis para construção de um indicador. Os dados foram normalizados em função do número de ligações de eletricidade de cada localidade com sistema isolado utilizando a função de Distribuição Normal- NORM. DIST(Dado,Média, Desvio Padrão) (MONTGOMERY, 2012) \& (OPENOFFICE, 2017). O Indicador Geral de Sustentabilidade (IGS) de um tema (Ambiental - A , Econômico - E e Social - S) foi obtido pela média dos indicadores individuais. Os indicadores individuais de um tema que destacam a baixa sustentabilidade do empreendimento receberam pesonulo no cálculo. Amaior sustentabilidade de um indicador recebeu valor máximo igual a 1. Ao final, um Índice de Sustentabilidade (IS) foi obtido pela média dos IGS (YADOO; CRUICKSHANK, 2012); (GARWOOD et al., 2012).

Os indicadores de sustentabilidade desta pesquisa estão descritos nas Tabelas dos Apêndice A, B e C.

Tabela 1 - Classificação de Sustentabilidade

\begin{tabular}{|c|c|}
\hline Índice de Sustentabilidade (IS) & Nível \\
\hline $0,8<$ IS & Alta \\
\hline $0,6<$ IS $\leq 0,8$ & Média \\
\hline IS $\leq 0.6$ & Baixa \\
\hline
\end{tabular}

Fonte: Adaptado de CERON (2013), EPE (2013), NBR-ISO (2007), CERON (2013), EPE (2013), MTE (2017), SEPOG-RO (2012), INEP (2012). 
Para cada indicador existe um objetivo específico, que pretende mensurar o impacto das variáveis de sustentabilidade dos sistemas micrigrids (LIU, 2014).

A hipótese nula $\left(H_{0}\right)$ considera a sustentabilidade do sistema microgrid, isto é, um valor esperado de IS igual a 1, maior valor absoluto possível neste modelo.
Não alcançando esse valor, hipótese alternativa $\left(H_{1}\right)$ será considerada e será possível classificar o sistema em três níveis de sustentabilidade (Tabela 1). Este teste corresponde às etapas de avaliação e ponderação propostos na metodologia sugerida por Liu (2014).

Figura 6 - Usina Térmica de Vista Alegre do Abunã

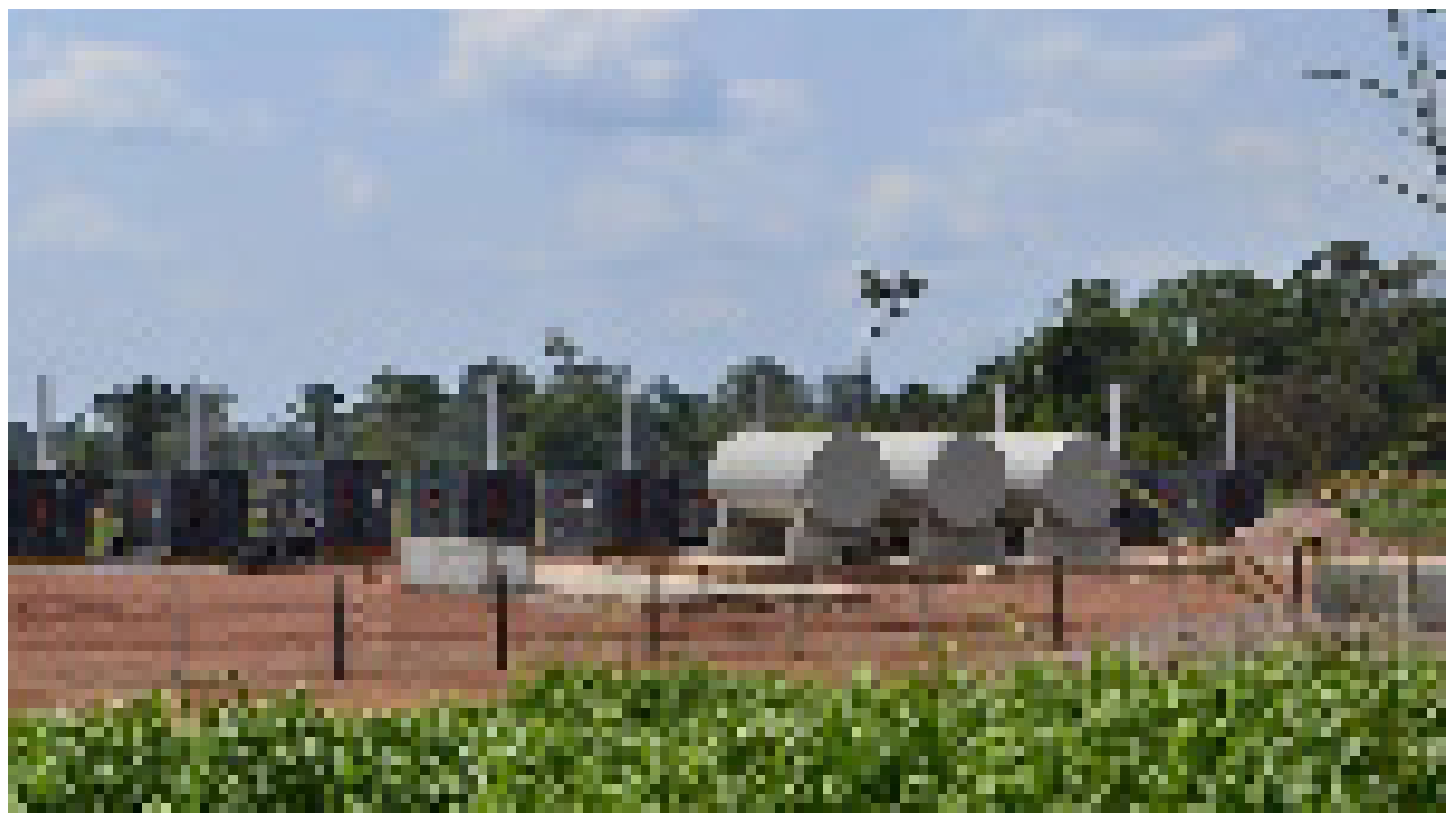

Fonte: Adaptado de Donizete (2015)

A classificação também pode ser avaliada em modo gráfico, indicado quais temas contribuem para maior ou menor eficiência do sistema. Nesta última etapa é realizada a agregação dos indicadores, conforme proposto por Liu (2014), em um indicador geral de sustentabilidade.

\section{Apresentação e discussão dos resultados}

O sistema isolado em Rondônia é formado por microgrids que possuem a geração diesel e é operado por duas empresas concessionárias: Rovema Energia e Brasil Bio Fuels (BBF). A Rovema Energia opera 15 unidades e a BBF é responsável por 10 usinas do Sistema Isolado. As UTEs foram instaladas de maneira semelhante, com grupos geradores dispostos dentro de contêineres (Figura 6).
As equipes de manutenção estão sediadas em Porto Velho (Rovema) e Ji-Paraná (BBF). De acordo com o site das empresas, a estrutura metálica é revestida com espuma termoacústica que é capaz de atenuar os ruídos do motor do equipamento. Em entrevista com as equipes de manutenção das concessionárias foi apurado que os equipamentos produzem ruídos de cerca de 55 a $75 \mathrm{~dB}$ quando estão na sua carga máxima. Os motores estão instalados em uma estrutura com revestimento acústico em contêineres (AGREKKO, 2017).

A água utilizada é fornecida pela concessionária local e, quando a rede não está disponível, são utilizados poços amazônicos. A água descartada é enviada para fossas sépticas. 0 tanque de combustível e bacia de contenção possui capacidade para até $110 \%$ de todo o volume de fluidos do gerador, incluindo o diesel. Em cada uma das unidades é gerado cerca de uma tonelada de lixo sólido e líquido, que são recolhidos para descarte 
adequado. De acordo com a equipe de manutenção, cerca de 15 pessoas contrata-das diretamente em cada empresa para atender todas as usinas, os sistemas são avaliados trimestralmente para monitoramento do impacto, manutenção preventiva e, quando necessário, de maneira corretiva (GPERS, 2017).

Segundo o representante da concessionária, existem planos de ação, mitigação, recuperação e impacto, entretanto, não são públicos. Essas informações são estratégicas para execução da concessão, uma vez que compõe a planilha de custos para prestação do serviço. Além disso, de acordo com os editais de licitação da ANEEL, não há referências para tais projetos para execução dos serviços de geração de energia, apenas a obtenção das licenças ambientais.

A ausência de divulgação dos dados sobre o plano de recuperação de área, plano de ação, mitigação de riscos, resiliência e impacto do monitoramento do empreendimento resultaram em um número nulo para alguns indicadores ambientais. Essas informações também não estão detalhadas no demonstrativo anual da CERON/ELETROBRAS (CERON/ELETROBRAS, 2016).

De acordo com documentação pública da CERON, gestora do sistema isolado, existe a previsão de interligação gradual de todo o sistema isolado. A princípio, as obras com recursos da CCC devem atender as localidades com maior número de habitantes.

Os resultados obtidos a partir da análise dos dados referentes aos sistemas isolados de Rondônia foram trabalhados estatisticamente e seguem metodologia proposta por Liu (2014). Contudo, não foi aplicada a lógica Fuzzy, pois os dados foram normalizados a partir dos valores médios e desvios padrão em função do número de ligações de energia em cada localidade. Esta alteração na metodologia proposta por Liu (2014) apresenta uma inovação que destaca as localidades com sistemas isolados, enfatizando o número de famílias atendidas em uma área. A quantidade de ligações de energia de uma localidade descreve a abrangência, a amplitude e capilaridade do sistema de distribuição de energia nas áreas isoladas. Esses dados correlacionados com indicadores próprios da região Amazônica descrevem a sustentabilidade dos sistemas microgrids com maior precisão.

A maior nota foi indicada de acordo com a contribuição do dado no desenvolvimento local (etapas de avaliação e ponderação) - (LIU, 2014). Por exemplo, é desejável que a taxa de mortalidade infantil seja nula, portanto, a localidade com a maior taxa observada recebeu nota 0 e a menor 1,0 . Os valores intermediários foram normalizados utilizando a função de distribuição Normal (NORM.DIST) (OPENOFFICE, 2017). Os investimentos em educação ambiental, ao contrário, devem ser maximizados e, neste caso, quanto maior o valor, mais próximo de 1,0 será o dado classificado. 0 indicador de sustentabilidade classificado com nível 1,0 representa a nota mais próxima do desejável. A nota máxima, mínima e intermediária estão correlacionadas em função do número de ligações em cada localidade (Unidades Consumidoras). Os dados brutos e indicadores deste estudo foram agregados e estão descritos nos Apêndices A, B e C. Por sua vez, o resultado do processamento dos dados e resultados estão dispostos na Figura 7 a seguir: 
Figura 7 - Resultados dos Indicadores de Sustentabilidade

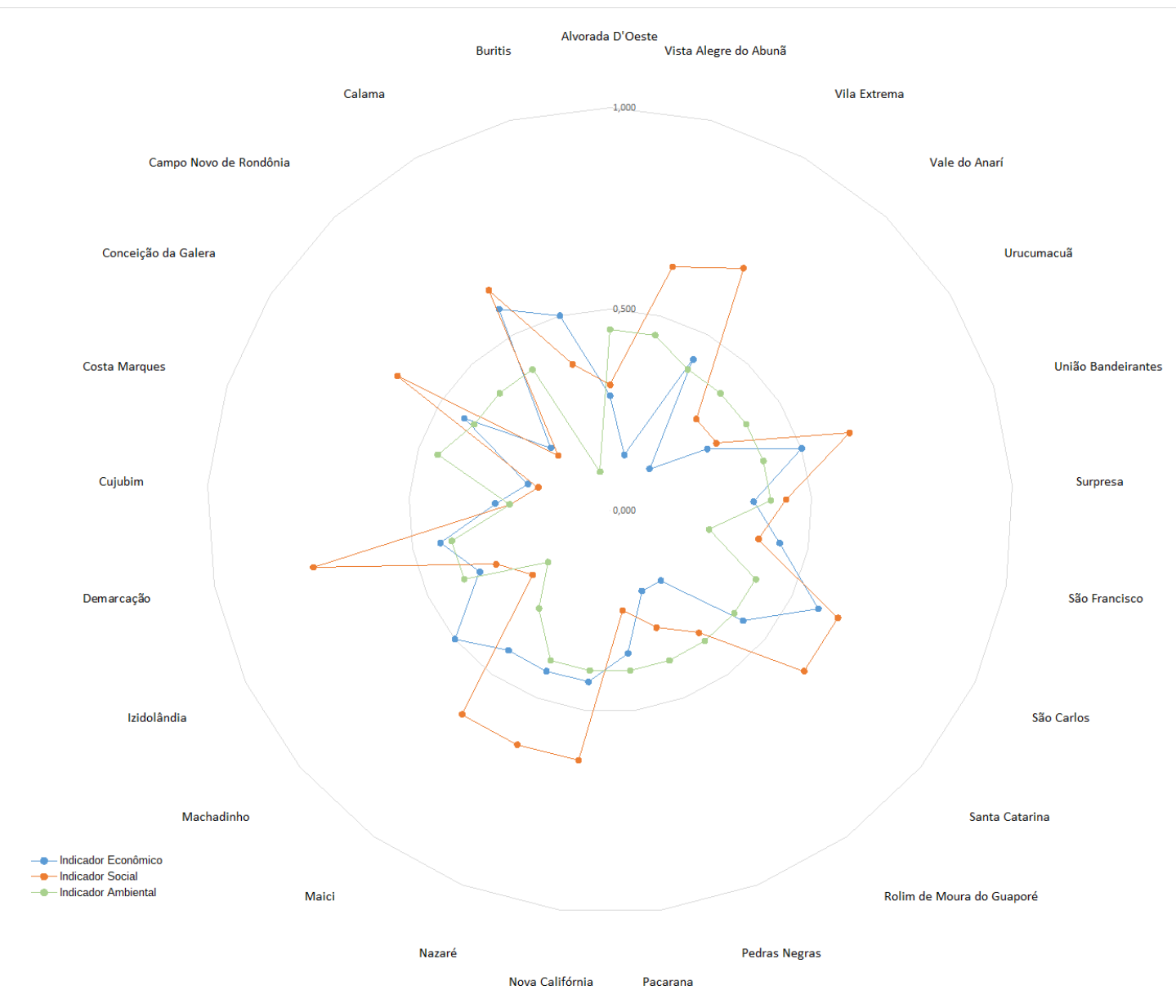

Fonte: Autor

Os indicadores econômicos apresentaram resultados com valores absolutos maiores que os demais indicadores avaliados neste estudo. Isso ocorreu porque os dados econômicos dos sistemas isolados estão delimitados pelos municípios, uma vez que houve restrição ao acesso de microdados que descrevem a realidade dos distritos onde estão estabelecidos os sistemas isolados. Os resultados obtidos descrevem uma realidade econômica média de cada município. Em 2015, a concessionária de energia de Rondônia realizou levantamento para fazer um diagnóstico socioeconômico, análise de demanda, análise de potencial energético renovável e georreferenciamento de comunidades remotas em regiões isoladas e investiu $\mathrm{R} \$$ 431.090,00 neste trabalho. Os resultados deste levantamento também sugerem as localidades destacadas neste trabalho. (CERON/ELETROBRAS, 2014).
Em relação aos dados econômicos, os sistemas isolados de Conceição da Galera; Demarcação; Izidolândia; Maici e Santa Catarina apresentaram os menores indicadores. Isso significa dizer que nas localidades onde esses sistemas estão instalados ocorreram investimentos elevados para implantação do sistema, mas não houve ampliação do número de ligações de energia, e ainda, uma resposta satisfatória em relação aos empregos formais criados, bem como investimentos em tecnologia que não reduziram do custo da energia fornecida.

A análise comparativa dos indicadores econômicos dos sistemas isolados pode ser avaliada na matriz de sustentabilidade na Figura 7. Os sistemas isolados que possuem os menores indicadores econômicos estão indicados ao centro do gráfico com pontos na cor azul. Os sistemas isolados com os melhores indicadores econômicos estão localizados mais a borda do gráfico. 
Figura 8 - Indicadores Gerais de Sustentabilidade dos Sistemas Isolados

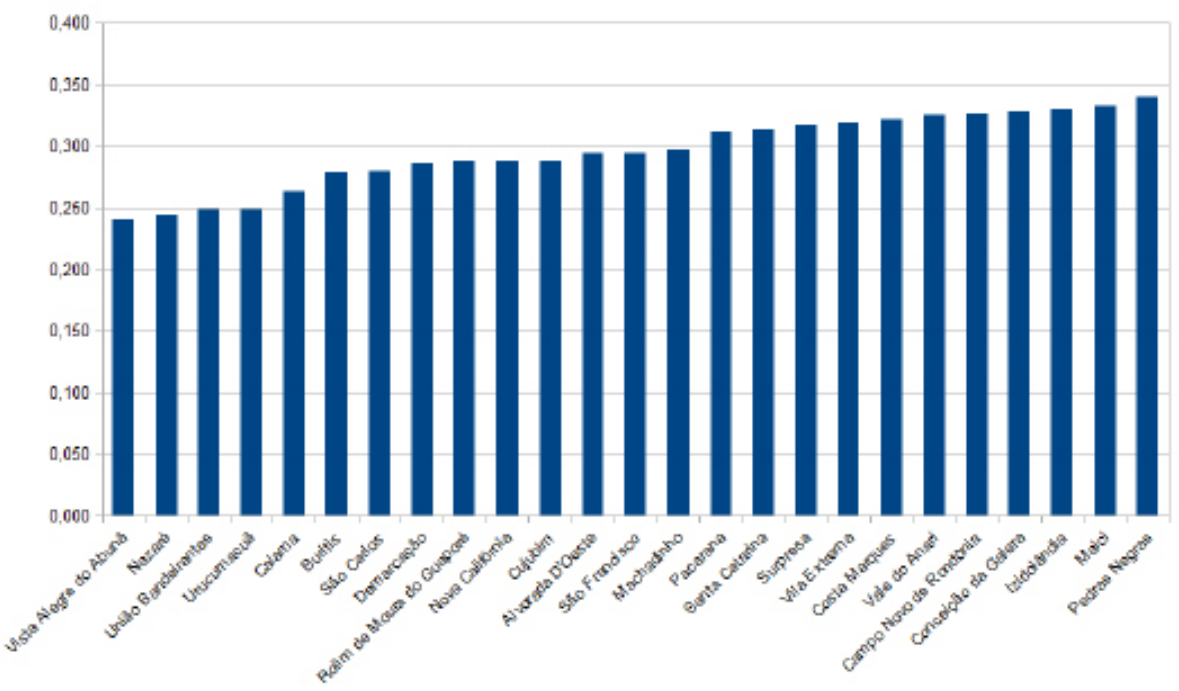

Fonte: Autor

Também é importante destacar os sistemas isolados que estão instalados em áreas com os menores indicadores sociais avaliados: Alvorada D'Oeste; Calama; Nazaré; São Carlos; Vista Alegre do Abunã. Nessas localidades é observado, de modo simultâneo, altos índices de malária, mortalidade infantil, bem como a menor oferta de serviços públicos essenciais, tais como número de ligação de energia, estabelecimentos do Sistema Único de Saúde (SUS) e unidades de ensino. Para essas localidades também é observada a maior população indígena residente. Essas comunidades estão a margem do desenvolvimento da Amazônia e estão sujeitas a grandes desafios para subsistência.

A análise comparativa dos indicadores sociais dos sistemas isolados está disposta na matriz de sustentabilidade na Figura 7 . Os sistemas isolados que possuem os menores indicadores sociais estão indicados ao centro do gráfico com pontos na cor laranja. Os sistemas isolados com os melhores indicadores sociais estão localizados mais a borda do gráfico.

Os indicadores ambientais não apresentaram resultados satisfatórios, ao contrário, apresentaram uma realidade preocupante. A estimativa de emissão de Gases Efeito Estufa (GEE), bem como o pequeno investimento em socioambiental não sustetam a manutenção deste modelo de geração de energia nas áreas isoladas. Agravando este cenário, as empresas concessionárias e prestadoras de serviços não publicam todos os dados relativos aos seus programas de monitoramento ambiental, planos de mitigação e prevenção adotados nas usinas térmicas do sistema isolado.

A análise comparativa dos indicadores ambientais dos sistemas isolados pode ser avaliada na matriz de sustentabilidade na Figura 7. Os sistemas isolados que possuem os menores indicadores ambientais estão indicados ao centro do gráfico com pontos na cor verde. Os sistemas isolados com os melhores indicadores ambientais estão localizados mais a borda do gráfıco.

A análise dos indicadores ambiental, social e econômico elaborados demonstram a baixa sustentabilidade das usinas de geração de energia dos sistemas isolados de Rondônia. A comparação dos índices de sustentabilidade dos sistemas isolados pode ser avaliada no gráfico da Figura 8. Todos os sistemas foram classificados com um índice de baixa sustentabilidade, uma vez que não contribuem de maneira significativa para o desenvolvimento ambiental, social e econômico. Os sistemas isolados de Calama; Demarcação; Nazaré; União Bandeirante; Urucumacuã; Vista Alegre do Abunã apresentaram os resultados mais expressivos de baixa sustentabilidade do empreendimento. 
De acordo com o teste de hipótese formulado neste trabalho, a hipótese nula $\left(H_{0}\right)$, que considera a sustentabilidade dos sistemas microgrids, não foi confirmada, isto é, o valor esperado do índice de Sustentabilidade (IS) não foi a 1, maior valor absoluto possível neste modelo. Em direção oposta, o IS foi bem diferente, recomendando a aceitação da hipótese alternativa $\left(H_{1}\right)$ que considera a baixa sustentabilidade dos sistemas isolados de Rondônia, uma vez que apresentaram valores baixos para o indicador geral (Figura 8).

\section{Considerações Finais}

Neste trabalho foi possível verificar que os sistemas isolados instalados em Rondônia não utilizam soluções sustentáveis para geração de energia elétrica. Essa avaliação considera os resultados obtidos na mensuração da sustentabilidade nas dimensões ambiental, econômica e social.

A metodologia utilizada para medir a sustentabilidade dos sistemas isolados demonstrou a baixa eficiência do modelo de geração de energia que utiliza geradores a diesel nas localidades mais distantes do estado de Rondônia.

O estudo demonstrou que o grau de sustentabilidade dos sistemas isolados muito baixo e não integra soluções sustentáveis nas microgrids rondonienses. Existem alternativas sustentáveis para geração de energia em Rondônia, contudo, não são incentivadas para uso nas comunidades isoladas. Os dados demonstraram que o combustível utilizado para geração de energia não fomenta o desenvolvimento nas localidades isolados, pode agredir o meio ambiente e não é capaz de integrar a micro/minigeração de fontes alternativas de energia elétrica. Este trabalho também demonstrou que a metodologia utilizada apresenta-se adequada para análise da sustentabilidade dos sistemas microgrids, em especial, no estado de Rondônia. 


\section{Referências}

AFGANA, N. H.; CARVALHOA, M. G.; HOVANOVB, N. V. (2000) Energy system assessment with sustainability indicators. Energy Policy, v. 28, n. 9, p. 603-612. Disponível em: <http://www.sciencedirect.com/science/article/pii/ S0301421500000458>. Acesso em: 01 ago. 2017.

AGREKKO. (2017) Geradores a diesel - Contêiner. Disponível em: <http://www.aggreko.com.br/produtos-servicos/ aluguel-geradores-energia/aluguel-geradores-a-diesel/>. Acesso em: 03 jul. 2017.

ANEEL (2016) Banco de Informações de Geração: Resumo Estadual. 2016. Disponível em: <http://www.aneel.gov.br/ area.cfm?idArea=15\&idPerfil=2\&idiomaAtual=0>. Acesso em: 29 ago. 2016.

BASF (2016). AgBalance - Measuring agricultural sustainability. Disponível em: <http://www.agro.basf.com/agr/APInternet/en/content/sustainability/measuring_sustainability/>. Acesso em: 29 nov. 2016.

CERON (2013). Projeto de Referência para Atendimento dos Sistemas Isolados com Previsão de Interligação da Eletrobras Distribuição Rondônia. Disponível em: <http://www2.aneel.gov.br/area.cfm?idArea=17\&idPerfil=8>. Acesso em: 03 jul. 2017.

CERON/ELETROBRAS (2016). Relatório de Demonstrações Financeiras e Administração. Disponível em: <http://www. diof.ro.gov.br/data/uploads/2016/04/DOE-15_04_2016.pdf>. Acesso em: 29 ago. 2016.

DONIZETE, J (2015). Rovema energia inicia obras para novos motores geradores. Disponível em: <http://maoamigaanuncios.com.br/rovema-energia-inicia-obras-para-novos-motores-geradores-na-ponta-do-abuna/>. Acesso em: 03 jul. 2017.

ELETROBRAS (2015). Plano anual de operações dos sistemas isolados para 2016. Grupo Técnico Operacional da Região Norte - GTON. Disponível em: <https://www.eletrobras.com>. Acesso em: 29 ago. 2016.

EPE (2013) Informe Técnico EPE-DEE-IT-021/2013-ro. Projeto de Referência para atendimento a dezesseis Sistemas Isolados de Rondônia, com previsão de interligação. Disponível em: <http://www2.aneel.gov.br/area. cfm?dArea=17\&idPerfil=8>. Acesso em: 03 jul. 2017.

FREEMAN, E. (1984) Strategic management: a stakeholder approach. Boston: Pitman. 292 p.

GARWOOD, A. et al. (2012) Evaluating and comparing three community small-scale wind electrification projects. Renewable and Sustainable Energy Reviews, v. 16, n. 7, p. 5379-5390, setembro 2012. Disponível em: <http://www. sciencedirect.com/science/article/pii/S1364032112002730 >. Acesso em: 01 ago. 2017.

GPERS (2017). Entrevista com a equipe de manutenção do Grupo Rovema em 06 de abril de 2017. Porto Velho: Grupo de Pesquisa Energia Renovável Sustentável.

HARVEY, D. O (2003). Novo Imperialismo. São Paulo: Editora Loyola. 140 p.

HATZIARGYRIOU, N. D.; VASILJEVSKA, J.; TSIKALAKIS, A. G. (2009) Quantification of economic, environmental and operational benefits of microgrids. IEEE Conference PowerTech, Bucareste, outubro 2009. Disponível em: <http:// ieeexplore.ieee.org/document/5281860/?part=1>. Acesso em: 01 ago. 2017.

HUBBARD, G. Measuring organizational performance: Beyond the triple bottom line. Business Strategy and Environment, v. 18, dezembro 2009. Disponível em: <http://onlinelibrary.wiley.com/doi/10.1002/bse.564/epdf>. Acesso em: 01 ago. 2017.

INEP (2012) Indicadores Educacionais - Resultados da Amostra. Disponível em:<http://portal.inep.gov.br/indicadoreseducacionais>. Acesso em: 03 jul. 2017.

KAPLAN, R. S.; NORTON, D. P. (1997) Estratégia em Ação: Balanced Scorecard. Rio de Janeiro: Elsevier. 231 p.

LILLO, P. et al. (2015) Assessing management models for off-grid renewable energy electrification projects using the

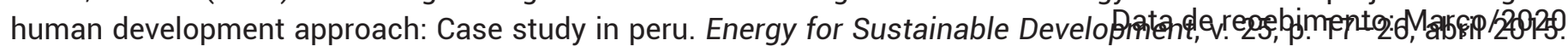

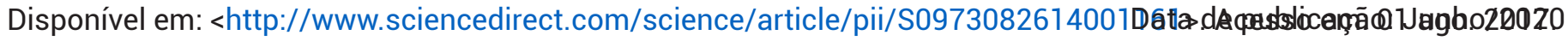


LIU, G. (2014) Development of a general sustainability indicator for renewable energy systems: A review. Renewable and Sustainable Energy Reviews, v. 31, n. 3, p. 611-621, março 2014. Disponível em: <http://www.sciencedirect.com/ science/article/pii/S1364032113008526>. Acesso em: 01 ago. 2017.

MME. (2016) Informações sobre o Programa Luz para Todos. Disponível em: <https://www.mme.gov.br/luzparatodos/ Asp/o_programa.asp>. Acesso em: 29 nov. 2016.

MONTGOMERY, D. C. (2012) Estatística Aplicada e Probabilidade Para Engenheiros. Rio de Janeiro: Brasiliense. MORET, A. S. (2014). Rondônia: 2000-2013. São Paulo: Fundação Perseu Abramo. 116 p.

MTE (2017). Indicadores do Ministério do Trabalho e Emprego. Disponível em: <http://bi.mte.gov.br/bgcaged/caged_ perfil_municipio/index.php>. Acesso em: 03 jul. 2017.

NASCIMENTO, E. (2012) Trajetória da sustentabilidade: do ambiental ao social, do social ao econômico. Dossiê Sustentabilidade, v. 26, n. 74, p. 51-64, 2012. Disponível em: <http://www.scielo.br/pdf/ea/v26n74/a05v26n74.pdf>. Acesso em: 01 ago. 2017.

NBR-ISO (2007). ISO 14.064 - Gases de efeito estufa - Parte 2: Especificação e orientação a organizações para a quantificação e elaboração de relatórios de emissões e remoções de gases de efeito estufa. Disponível em: <http:// abnt.org.br/paginampe/biblioteca/files/upload/anexos/pdf/4ee5b810af4a3aee073ab89f0a573a1a.pdf>. Acesso em: 03 jul. 2017.

OPENOFFICE (2017). Documentation/How Tos/Calc: NORMDIST function. Disponível em: <https://wiki.openoffice. org/wiki/Documentation/How_Tos/Calc:_NORMDIST_function>. Acesso em: 03 jul. 2017.

OSTROM, E. (2009) A general framework for analyzing sustainability of social-ecological systems. Science, v. 325, n. 5939, p. 419-422, julho 2009. Disponível em: <http://www.scielo.br/pdf/ea/v26n74/a05v26n74.pdf>. Acesso em: 01 ago. 2017.

REIS, L. B. (2011). Geração de Energia Elétrica. Barueri: Manole. 203 p.

SEPOG-RO (2012). Rondônia em Números. Disponível em: <http://www.sepog.ro.gov.br/Conteudo/Exibir/273> Acesso em: 03 jul. 2017.

SLOUGH, T.; URPELAINEN, J.; YANG, J. (2015) Light for all? evaluating brazil's rural electrification progress 2000-2010. Energy Policy, v. 86, p. 315-327, novembro 2015. Disponível em: <http://www.sciencedirect.com/science/article/pii/ S0301421515300124>. Acesso em: 01 ago. 2017.

TOLMASQUIN, M. T. (2016). Energia Renovável: Hidráulica, Biomassa, Eólica, Solar, Oceânica. Rio de Janeiro: EPE. Disponível em: <http://www.epe.gov.br/Documents/Energia\%20Renov\%C3\%A1vel\%20-\%200nline2016maio2016. pdf>. Acesso em: 29 ago. 2016.

YADOO, A.; CRUICKSHANK, H. (2012) The role for low carbon electrificationtechnologies in poverty reduction and climate change strategies: A focus on renewable energy mini-grids with case studies in nepal, peru and kenya. Energy Policy, v. 42, p. 591-602, março 2012. Disponível em: <http://www.sciencedirect.com/science/article/pii/ S0301421511010354>. Acesso em: 01 ago. 2017. 
Apêndice A - Indicadores e Dados Ambientais dos Sistemas Microgrids Isolados de Rondônia

Apêndice A - Indicadores e Dados Ambientais dos Sistemas Microgrids Isolados de Rondônia

\begin{tabular}{|c|c|c|c|c|c|}
\hline Sistema Isolado & IA1 & IA2 & IA3 & IA4 & IA5 \\
\hline Indicador & $\begin{array}{c}\text { Emissão de CO2 } \\
\text { (Dióxido de Carbono) }\end{array}$ & $\begin{array}{l}\text { Emissão de CH4 } \\
\quad \text { (Metano) }\end{array}$ & $\begin{array}{l}\text { Emissão de Nox } \\
\text { (Óxido de Nitrogênio) }\end{array}$ & $\begin{array}{c}\text { Emissão SO2 } \\
\text { (Dióxido de Enxofre) }\end{array}$ & $\begin{array}{l}\text { Investimentos em Ações } \\
\text { Socioambientais }\end{array}$ \\
\hline Unidade & Ton/ano & Ton/ano & Ton/ano & Ton/ano & Reais \\
\hline Alvorada D'Oeste & $14.685,11$ & 6,14 & 0,11 & 0,22 & $429.769,24$ \\
\hline Buritis & $56.975,42$ & 23,83 & 0,43 & 0,85 & $1.039 .700,00$ \\
\hline Calama & $2.096,35$ & 0,88 & 0,02 & 0,03 & $51.983,38$ \\
\hline $\begin{array}{l}\text { Campo Novo de } \\
\text { Rondônia }\end{array}$ & $5.662,98$ & 2,37 & 0,04 & 0,08 & $214.884,62$ \\
\hline Conceição da Galera & 103,16 & 0,04 & 0,00 & 0,00 & $5.198,34$ \\
\hline Costa Marques & $8.250,66$ & 3,45 & 0,06 & 0,12 & $429.769,24$ \\
\hline Cujubim & $23.407,64$ & 9,79 & 0,18 & 0,35 & $693.133,33$ \\
\hline Demarcação & 225,39 & 0,09 & 0,00 & 0,00 & $5.198,34$ \\
\hline Izidolândia & 833,11 & 0,35 & 0,01 & 0,01 & $43.723,87$ \\
\hline Machadinho & $39.418,73$ & 16,49 & 0,30 & 0,59 & $808.655,56$ \\
\hline Maici & 59,13 & 0,02 & 0,00 & 0,00 & $5.198,34$ \\
\hline Nazaré & 853,53 & 0,36 & 0,01 & 0,01 & $51.983,38$ \\
\hline Nova Califórnia & $6.401,32$ & 2,68 & 0,05 & 0,10 & $191.477,78$ \\
\hline Pacarana & $2.578,35$ & 1,08 & 0,02 & 0,04 & $144.444,96$ \\
\hline Pedras Negras & 187,64 & 0,08 & 0,00 & 0,00 & $5.198,34$ \\
\hline $\begin{array}{l}\text { Rolim de Moura do } \\
\text { Guaporé }\end{array}$ & 612,22 & 0,26 & 0,00 & 0,01 & $5.198,34$ \\
\hline Santa Catarina & 185,38 & 0,08 & 0,00 & 0,00 & $5.198,34$ \\
\hline São Carlos & $1.524,47$ & 0,64 & 0,01 & 0,02 & $51.983,38$ \\
\hline $\begin{array}{l}\text { São Francisco do } \\
\text { Guaporé }\end{array}$ & $21.940,48$ & 9,18 & 0,17 & 0,33 & $554.653,87$ \\
\hline Surpresa & 789,49 & 0,33 & 0,01 & 0,01 & $10.396,68$ \\
\hline União Bandeirantes & $9.396,46$ & 3,93 & 0,07 & 0,14 & $136.769,84$ \\
\hline Urucumacuã & $1.095,71$ & 0,46 & 0,01 & 0,02 & $32.792,91$ \\
\hline Vale do Anarí & $8.058,41$ & 3,37 & 0,06 & 0,12 & $300.838,47$ \\
\hline Vila Extrema & $6.543,72$ & 2,74 & 0,05 & 0,10 & $257.861,55$ \\
\hline Vista Alegre do Abunã & $15.560,33$ & 6,51 & 0,12 & 0,23 & $429.769,24$ \\
\hline
\end{tabular}


Apêndice B - Indicadores e Dados Econômicos dos Sistemas Microgrids Isolados de Rondônia

Apêndice B - Indicadores e Dados Econômicos dos Sistemas Microgrids Isolados de Rondônia

\begin{tabular}{|c|c|c|c|c|c|c|c|}
\hline Sistema Isolado & IE1 & IE2 & IE3 & IE4 & IE5 & IE6 & IE7 \\
\hline Indicador & Investimento & $\begin{array}{c}\text { Empregos } \\
\text { Formais }\end{array}$ & $\begin{array}{l}\text { Renda } \\
\text { per capta }\end{array}$ & $\begin{array}{l}\text { Ligações de } \\
\text { Eletricidade }\end{array}$ & $\begin{array}{l}\text { Custo da } \\
\text { Tecnologia }\end{array}$ & $\begin{array}{l}\text { Custo da } \\
\text { Eletricidade }\end{array}$ & $\begin{array}{l}\text { Custo de } \\
\text { Impacto }\end{array}$ \\
\hline Unidade & Reais & Unidade & $\begin{array}{c}\text { Reais/Habitant } \\
\mathrm{e}\end{array}$ & Unidade & Reais & Reais & Reais \\
\hline Alvorada D'Oeste & $11.961 .335,17$ & 686 & $11.847,00$ & 5.064 & $4.777 .461,69$ & 220,04 & 2.362 \\
\hline Buritis & $29.057 .884,73$ & 2.737 & $12.935,00$ & 10.272 & $11.436 .700,00$ & 157,14 & 2.829 \\
\hline Calama & $1.452 .849,42$ & 100.426 & $23.639,00$ & 350 & $571.817,20$ & 225,49 & 4.151 \\
\hline Campo Novo de Rondônia & $6.005 .667,58$ & 324 & $14.830,00$ & 2.287 & $2.363 .730,85$ & 243,47 & 2.626 \\
\hline Conceição da Galera & $833.605,62$ & 100.426 & $23.639,00$ & 28 & $400.272,04$ & 294,03 & 29.772 \\
\hline Costa Marques & $12.011 .335,17$ & 442 & $10.572,00$ & 2.373 & $4.727 .461,69$ & 220,04 & 5.062 \\
\hline Cujubim & $19.371 .923,15$ & 1.057 & $12.287,00$ & 4.320 & $7.624 .466,67$ & 192,41 & 4.484 \\
\hline Demarcação & $833.605,62$ & 100.426 & $23.639,00$ & 37 & $400.272,04$ & 282,47 & 22.530 \\
\hline Izidolândia & $1.222 .009,52$ & 1.970 & $14.777,00$ & 435 & $480.962,61$ & 290,00 & 2.809 \\
\hline Machadinho & $22.600 .577,01$ & 2.035 & $11.875,00$ & 8.924 & $8.895 .211,11$ & 192,41 & 2.533 \\
\hline Maici & $833.605,62$ & 100.426 & $23.639,00$ & 15 & $400.272,04$ & 294,03 & 55.574 \\
\hline Nazaré & $1.452 .849,42$ & 100.426 & $23.639,00$ & 105 & $571.817,20$ & 229,65 & 13.837 \\
\hline Nova Califórnia & $5.351 .485,15$ & 100.426 & $23.639,00$ & 1.233 & $2.106 .255,53$ & 243,47 & 4.340 \\
\hline Pacarana & $4.036 .995,69$ & 4.279 & $15.551,00$ & 559 & $1.588 .894,35$ & 25,00 & 7.222 \\
\hline Pedras Negras & $833.605,62$ & 977 & $14.637,00$ & 25 & $400.272,04$ & 294,03 & 33.344 \\
\hline Rolim de Moura do Guaporé & $1.233 .357,91$ & 1.970 & $14.777,00$ & 128 & $441.858,75$ & 240,31 & 9.636 \\
\hline Santa Catarina & $833.605,62$ & 100.426 & $23.639,00$ & 43 & $400.272,04$ & 279,35 & 19.386 \\
\hline São Carlos & $1.452 .849,42$ & 100.426 & $23.639,00$ & 302 & $571.817,20$ & 217,79 & 4.811 \\
\hline São Francisco do Guaporé & 18.107.002,74 & 977 & $14.637,00$ & 4.582 & $7.091 .192,54$ & 202,85 & 3.952 \\
\hline Surpresa & $1.254 .150,89$ & 2.840 & $14.448,00$ & 240 & $452.255,43$ & 252,80 & 5.226 \\
\hline União Bandeirantes & $3.822 .489,40$ & 100.426 & $23.639,00$ & 1.287 & $1.504 .468,23$ & 247,02 & 2.970 \\
\hline Urucumacuã & $916.507,13$ & 10.056 & $22.897,00$ & 84 & $360.721,96$ & 290 & 10.911 \\
\hline Vale do Anarí & $8.407 .934,62$ & 278 & $9.849,00$ & 3.054 & $3.309 .223,18$ & 243,47 & 2.753 \\
\hline Vila Extrema & $7.206 .801,10$ & 100.426 & $23.639,00$ & 2.123 & $2.836 .477,01$ & 248,88 & 3.395 \\
\hline Vista Alegre do Abunã & $12.011 .335,17$ & 1.970 & $14.777,00$ & 1.104 & $4.727 .461,69$ & 208,65 & 10.880 \\
\hline
\end{tabular}


Apêndice C - Indicadores e Dados Sociais dos Sistemas Microgrids Isolados de Rondônia

Apêndice C - Indicadores e Dados Sociais dos Sistemas Microgrids Isolados de Rondônia

\begin{tabular}{|c|c|c|c|c|c|c|c|c|}
\hline Sistema Isolado & IS1 & IS2 & IS3 & IS4 & IS5 & IS6 & IS7 & IS8 \\
\hline Indicador & $\begin{array}{l}\text { Estabelecimentos } \\
\text { de Saúde SUS }\end{array}$ & $\begin{array}{l}\text { Índice de } \\
\text { Malária }\end{array}$ & $\begin{array}{l}\text { Mortalidade } \\
\text { Infantil }\end{array}$ & $\begin{array}{l}\text { Escolas de } \\
\text { Ensino } \\
\text { Fundamental }\end{array}$ & $\begin{array}{c}\text { Ensino } \\
\text { Fundamental }\end{array}$ & $\begin{array}{l}\text { Ensino } \\
\text { Médio }\end{array}$ & $\begin{array}{c}\text { Sem instrução } \\
\text { e Fundamental } \\
\text { Incompleto }\end{array}$ & $\begin{array}{l}\text { População } \\
\text { Indígena }\end{array}$ \\
\hline Unidade & Unidade & $\%$ & $\%$ & Unidades & Unidades & Unidades & Unidades & Unidades \\
\hline Alvorada D'Oeste & 8 & 2,65 & 5,43 & 11 & 2.364 & 641 & 9.133 & 15 \\
\hline Buritis & 5 & 10,18 & 19,35 & 34 & 6.285 & 1.264 & 17.825 & 39 \\
\hline Calama & 99 & 36,13 & 13,36 & 232 & 80.208 & 17.083 & 162.564 & 1.411 \\
\hline Campo Novo de Rondônia & 3 & 23,91 & 11,05 & 8 & 2.308 & 411 & 7.507 & 9 \\
\hline Conceição da Galera & 99 & 36,13 & 13,36 & 232 & 80.208 & 17.083 & 162.564 & 1.411 \\
\hline Costa Marques & 9 & 14,91 & 18,73 & 10 & 2.846 & 569 & 7.283 & 224 \\
\hline Cujubim & 1 & 26,92 & 16,67 & 7 & 3.456 & 652 & 8.945 & 3 \\
\hline Demarcação & 99 & 36,13 & 13,36 & 232 & 80.208 & 17.083 & 162.564 & 1.411 \\
\hline Izidolândia & 7 & 1,65 & 8,8 & 34 & 3.944 & 1.018 & 13.470 & 436 \\
\hline Machadinho & 14 & 37,19 & 9,86 & 29 & 6.148 & 1.062 & 17.965 & 32 \\
\hline Maici & 99 & 36,13 & 13,36 & 232 & 80.208 & 17.083 & 162.564 & 1.411 \\
\hline Nazaré & 99 & 36,13 & 13,36 & 232 & 80.208 & 17.083 & 162.564 & 1.411 \\
\hline Nova Califórnia & 99 & 36,13 & 13,36 & 232 & 80.208 & 17.083 & 162.564 & 1.411 \\
\hline Pacarana & 9 & 2,11 & 22,22 & 23 & 4.958 & 1.305 & 15.087 & 391 \\
\hline Pedras Negras & 12 & 1,90 & 16,23 & 12 & 3.032 & 716 & 8.865 & 379 \\
\hline $\begin{array}{l}\text { Rolim de Moura do } \\
\text { Guaporé }\end{array}$ & 7 & 1,65 & 8,8 & 34 & 3.944 & 1.018 & 13.470 & 436 \\
\hline Santa Catarina & 99 & 36,13 & 13,36 & 232 & 80.208 & 17.083 & 162.564 & 1.411 \\
\hline São Carlos & 99 & 36,13 & 13,36 & 232 & 80.208 & 17.083 & 162.564 & 1.411 \\
\hline São Francisco do Guaporé & 12 & 1,90 & 16,23 & 12 & 3.032 & 716 & 8.865 & 379 \\
\hline Surpresa & 13 & 15,14 & 19,57 & 62 & 8.869 & 1.620 & 19.374 & 3.998 \\
\hline União Bandeirantes & 99 & 36,13 & 13,36 & 232 & 80.208 & 17.083 & 162.564 & 1.411 \\
\hline Urucumacuã & 6 & 1,29 & 23,45 & 21 & 5.768 & 1.450 & 15.517 & 331 \\
\hline Vale do Anarí & 1 & 16,93 & $\mathrm{~N} / \mathrm{E}$ & 7 & 1.621 & 247 & 5.533 & 6 \\
\hline Vila Extrema & 99 & 36,13 & 13,36 & 232 & 80.208 & 17.083 & 162.564 & 1.411 \\
\hline Vista Alegre do Abunã & 99 & 36,13 & 13,36 & 232 & 80.208 & 17.083 & 162.564 & 1.411 \\
\hline
\end{tabular}

